Advances in Fuzzy Systems - Applications and Theory - Vol. 24

\title{
Nonlinear Integrals and Their Applications in Data Mining
}




\section{ADVANCES IN FUZZY SYSTEMS - APPLICATIONS AND THEORY}

Honorary Editor: Lotfi A. Zadeh (Univ. of California, Berkeley)

Series Editors: Kaoru Hirota (Tokyo Inst. of Tech.),

George J. Klir (Binghamton Univ.-SUNY),

Elie Sanchez (Neurinfo),

Pei-Zhuang Wang (West Texas A\&M Univ.),

Ronald R. Yager (Iona College)

Published

Vol. 9: Fuzzy Topology

(Y. M. Liu and M. K. Luo)

Vol. 10: Fuzzy Algorithms: With Applications to Image Processing and Pattern Recognition

(Z. Chi, H. Yan and T. D. Pham)

Vol. 11: Hybrid Intelligent Engineering Systems

(Eds. L. C. Jain and R. K. Jain)

Vol. 12: Fuzzy Logic for Business, Finance, and Management

(G. Bojadziev and M. Bojadziev)

Vol. 13: Fuzzy and Uncertain Object-Oriented Databases: Concepts and Models (Ed. R. de Caluwe)

Vol. 14: Automatic Generation of Neural Network Architecture Using Evolutionary Computing

(Eds. E. Vonk, L. C. Jain and R. P. Johnson)

Vol. 15: Fuzzy-Logic-Based Programming (Chin-Liang Chang)

Vol. 16: Computational Intelligence in Software Engineering (W. Pedrycz and J. F. Peters)

Vol. 17: Nonlinear Integrals and Their Applications in Data Mining (Z. Y. Wang, R. Yang and K.-S. Leung)

Vol. 18: Factor Space, Fuzzy Statistics, and Uncertainty Inference (Forthcoming) (P. Z. Wang and X. H. Zhang)

Vol. 19: Genetic Fuzzy Systems, Evolutionary Tuning and Learning of Fuzzy Knowledge Bases

(O. Cordón, F. Herrera, F. Hoffmann and L. Magdalena)

Vol. 20: Uncertainty in Intelligent and Information Systems

(Eds. B. Bouchon-Meunier, R. R. Yager and L. A. Zadeh)

Vol. 21: Machine Intelligence: Quo Vadis?

(Eds. P. Sincák, J. Vascák and K. Hirota)

Vol. 22: Fuzzy Relational Calculus: Theory, Applications and Software (With CD-ROM)

(K. Peeva aňd Y. Kyošěv)

Vol. 23: Fuzzy Logic for Business, Finance and Management (2nd Edition) (G. Bojadziev and M. Bojadziev) 
Advances in Fuzzy Systems - Applications and Theory - Vol. 24

Nonlinear Integrals

and Their Applications

in Data Mining

\section{Zhenyuan Wang}

University of Nebraska at Omaha, USA

\section{Rong Yang}

Shen Zhen University, China

\section{Kwong-Sak Leung}

Chinese University of Hong Kong, China

\section{World Scientific}




\section{Published by}

World Scientific Publishing Co. Pte. Ltd.

5 Toh Tuck Link, Singapore 596224

USA office: 27 Warren Street, Suite 401-402, Hackensack, NJ 07601

UK office: 57 Shelton Street, Covent Garden, London WC2H 9HE

\section{British Library Cataloguing-in-Publication Data}

A catalogue record for this book is available from the British Library.

\section{NONLINEAR INTEGRALS AND THEIR APPLICATIONS IN DATA MINING Advances in Fuzzy Systems - Applications and Theory - Vol. 17 \\ Copyright (C) 2010 by World Scientific Publishing Co. Pte. Ltd. \\ All rights reserved. This book, or parts thereof, may not be reproduced in any form or by any means, electronic or mechanical, including photocopying, recording or any information storage and retrieval system now known or to be invented, without written permission from the Publisher.}

For photocopying of material in this volume, please pay a copying fee through the Copyright Clearance Center, Inc., 222 Rosewood Drive, Danvers, MA 01923, USA. In this case permission to photocopy is not required from the publisher.

ISBN-13 978-981-281-467-8

ISBN-10 981-281-467-1

Printed in Singapore by Mainland Press Pte Ltd. 
To our families

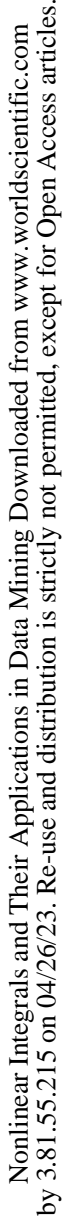


This page intentionally left blank 


\section{Preface}

The theory of nonadditive set functions and relevant nonlinear integrals, as a new mathematics branch, has been developed for more than thirty years. Starting from the beginning of the nineties of the last century, several monographs were published. The first author of this monograph and Professor George J. Klir (The State University of New York at Binghamton) have published two books, Fuzzy Measure Theory (Plenum Press, New York, 1992) and Generalized Measure Theory (Springer-verlag, New York, 2008) on this topic. These two books cover most of their theoretical research results with colleagues at the Chinese University of Hong Kong in the area of nonadditive set functions and relevant nonlinear integrals. Since the 1980s, nonadditive set functions and nonlinear integrals have been successfully applied in information fusion and data mining. However, only a few applications are involved in the above-mentioned books. As a supplement and indepth material, the current monograph, Nonlinear Integrals and Their Applications in Data Mining, concentrates on the applications in data analysis. Since the number of attributes in any database is always finite, we focus on our fundamentally theoretical discussion of nonadditive set function and nonlinear integrals, which are presented in the first several chapters, on the finite universal set, and abandon all convergence and limit theorems.

As for the terminology adopted in the current monograph, words like monotone measure is used for a set function that is nonnegative, monotonic, and vanishing at the empty set. It has no fuzziness in the meaning of Zadeh's fuzzy sets. Unfortunately, its original name is fuzzy measure in literature. Word "fuzzy" here is not proper. For example, 
words "fuzzy-valued fuzzy measure defined on fuzzy sets" causes confusion to some people. Such a revision is the same as made in book Generalized Measure Theory. However, in this monograph, we prefer to use efficiency measure to name a set function that is nonnegative and vanishing at the empty set, rather than using general measure. This is more convenient and intuitive, and leaves more space for further generalizing the domain or the range of the set functions. Hence, similar to the classical case in measure theory [Halmos 1950], the set functions that vanish at the empty set and may assume both nonnegative and negative real values are naturally named as signed efficiency measures. The signed efficiency measures were also called non-monotonic fuzzy measures by some scholars. Since, in general, the efficiency measures are non-monotonic too, to distinguish the set functions satisfying only the condition of vanishing at the empty set from the efficiency measures and to emphasize that they can assume both positive and negative values as well as zero, we prefer to use the current name, signed efficiency measures, for this type of set functions with the weakest restriction. Thus, in this monograph, we discuss and apply three layers of set functions named monotone measures, efficiency measures, and signed efficiency measures respectively.

The contents of this monograph have been used as the teaching materials of two graduate level courses at the University of Nebraska at Omaha since 2004. Also, some parts of this monograph have been provided to a number of master degree and Ph.D. degree graduate students in the University of Nebraska at Omaha, the University of Nebraska at Lincoln, the Chinese University of Hong Kong, and the Chinese Academy Sciences, for preparing their dissertations.

This monograph may benefit the relevant research workers. It is also possible to be used as a textbook of some graduate level courses for both mathematics and engineering major students. A number of exercises on the basic theory of nonadditive set functions and relevant nonlinear integrals are available in Chapters $2-5$ of the monograph.

Several former graduate students of the first author provided some algorithms, examples, and figures. We appreciate their valuable contributions to this monograph. We also thank the Department of Computer Science and Engineering of the Chinese University of Hong 
Kong, the Department of System Science and Industrial Engineering of the State University of New York at Binghamton and, especially, the Department of Mathematics, as well as the Art and Science College of the University of Nebraska at Omaha for their support and help.

Zhenyuan Wang Rong Yang Kwong-Sak Leung 
This page intentionally left blank 


\section{Contents}

Preface ............................................................................................................ vii

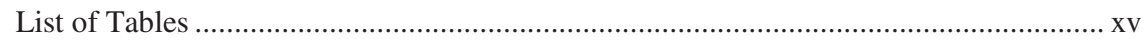

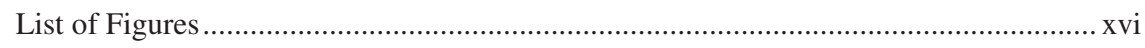

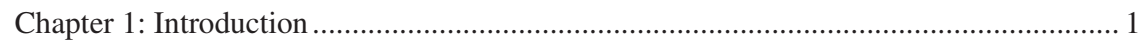

Chapter 2: Basic Knowledge on Classical Sets ............................................................... 4

2.1 Classical Sets and Set Inclusion ........................................................................... 4

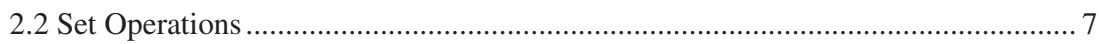

2.3 Set Sequences and Set Classes ...................................................................... 10

2.4 Set Classes Closed Under Set Operations ........................................................ 13

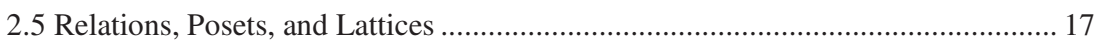

2.6 The Supremum and Infimum of Real Number Sets ......................................... 20

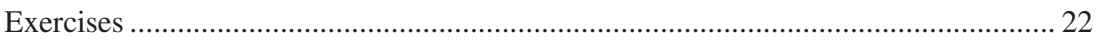

Chapter 3: Fuzzy Sets ............................................................................................ 24

3.1 The Membership Functions of Fuzzy Sets ........................................................ 24

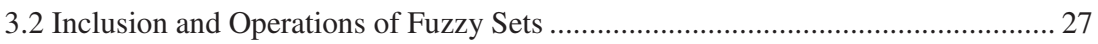

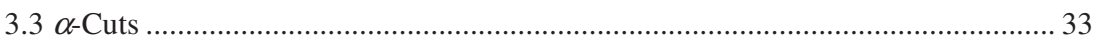

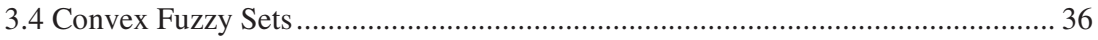

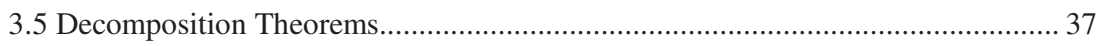

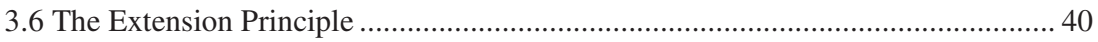

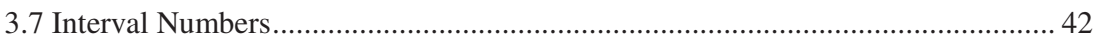

3.8 Fuzzy Numbers and Linguistic Attribute .......................................................... 45

3.9 Binary Operations for Fuzzy Numbers................................................................ 51

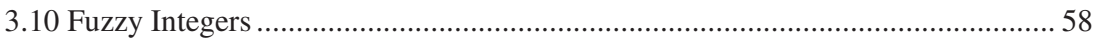

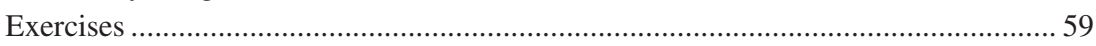

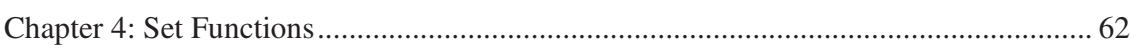

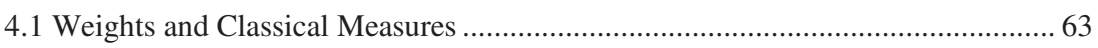

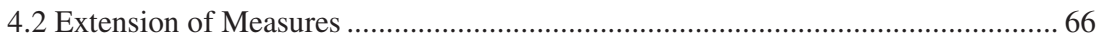

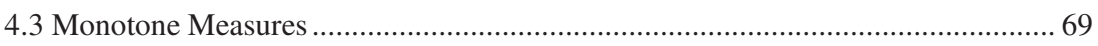

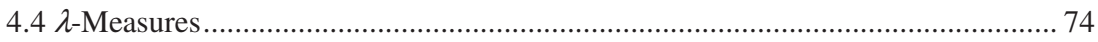




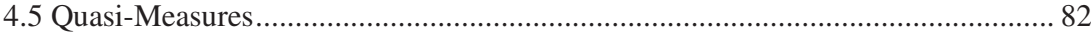

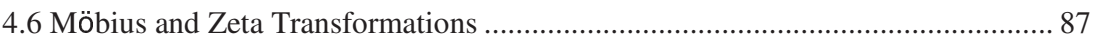

4.7 Belief Measures and Plausibility Measures ......................................................... 91

4.8 Necessity Measures and Possibility Measures ................................................. 102

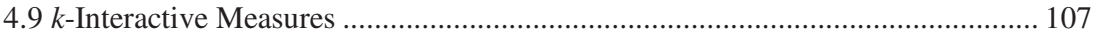

4.10 Efficiency Measures and Signed Efficiency Measures..................................... 108

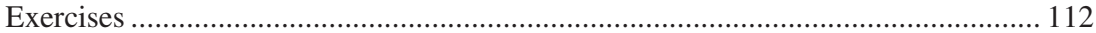

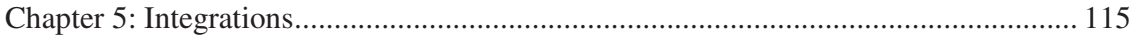

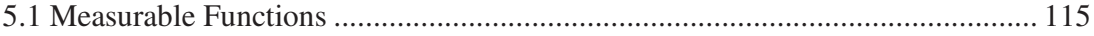

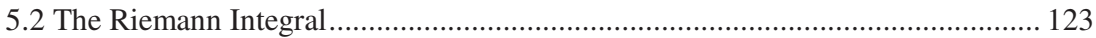

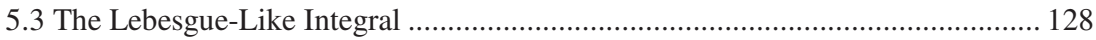

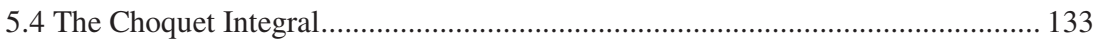

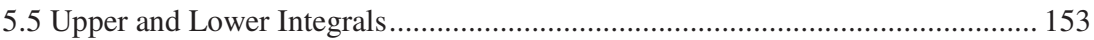

$5.6 r$-Integrals on Finite Spaces..................................................................... 162

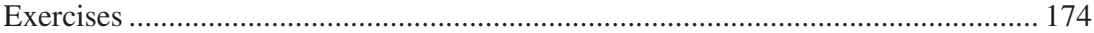

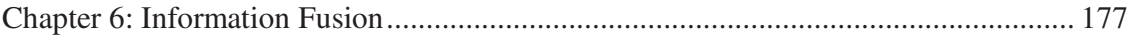

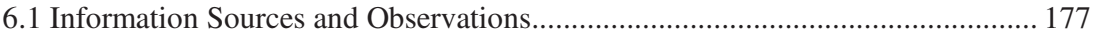

6.2 Integrals Used as Aggregation Tools ............................................................ 181

6.3 Uncertainty Associated with Set Functions ................................................... 186

6.4 The Inverse Problem of Information Fusion ....................................................... 190

Chapter 7: Optimization and Soft Computing....................................................... 193

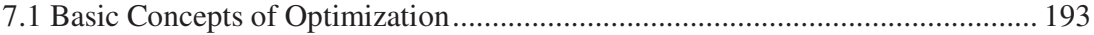

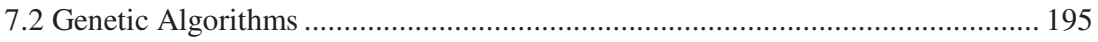

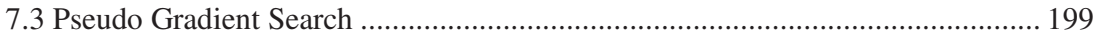

7.4 A Hybrid Search Method ................................................................................ 202

Chapter 8: Identification of Set Functions ................................................................... 204

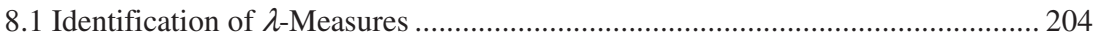

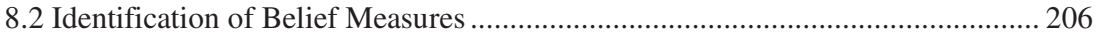

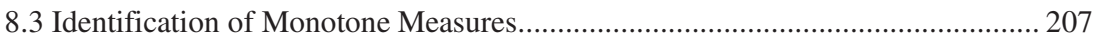

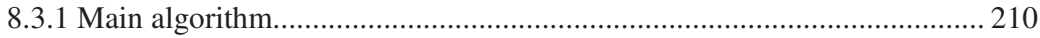

8.3.2 Reordering algorithm ......................................................................... 211

8.4 Identification of Signed Efficiency Measures by a Genetic Algorithm ............... 213

8.5 Identification of Signed Efficiency Measures by the Pseudo Gradient Search.... 215

8.6 Identification of Signed Efficiency Measures Based on the Choquet Integral

by an Algebraic Method........................................................................ 217

8.7 Identification of Monotone Measures Based on $r$-Integrals by a Genetic

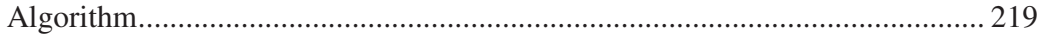

Chapter 9: Multiregression Based on Nonlinear Integrals ....................................... 221

9.1 Linear Multiregression ............................................................................. 221 
9.2 Nonlinear Multiregression Based on the Choquet Integral................................ 226

9.3 A Nonlinear Multiregression Model Accommodating Both Categorical and Numerical Predictive Attributes

9.4 Advanced Consideration on the Multiregression Involving Nonlinear

Integrals

9.4.1 Nonlinear multiregressions based on the Choquet integral with quadratic core.

9.4.2 Nonlinear multiregressions based on the Choquet integral involving unknown periodic variation

9.4.3 Nonlinear multiregressions based on upper and lower integrals ............... 236

Chapter 10: Classifications Based on Nonlinear Integrals .......................................... 238

10.1 Classification by an Integral Projection............................................................. 238

10.2 Nonlinear Classification by Weighted Choquet Integrals ............................... 242

10.3 An Example of Nonlinear Classification in a Three-Dimensional Sample Space.....

10.4 The Uniqueness Problem of the Classification by the Choquet Integral with a Linear Core

10.5 Advanced Consideration on the Nonlinear Classification Involving the

Choquet Integral

10.5.1 Classification by the Choquet integral with the widest gap between classes

10.5.2 Classification by cross-oriented projection pursuit ............................... 268

10.5.3 Classification by the Choquet integral with quadratic core .................... 270

Chapter 11: Data Mining with Fuzzy Data .................................................................. 272

11.1 Defuzzified Choquet Integral with Fuzzy-Valued Integrand (DCIFI).............. 273

11.1.1 The $\alpha$-level set of a fuzzy-valued function......................................... 274

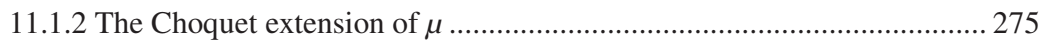

11.1.3 Calculation of DCIFI ................................................................... 277

11.2 Classification Model Based on the DCIFI........................................................ 282

11.2.1 Fuzzy data classification by the DCIFI .............................................. 283

11.2.2 GA-based adaptive classifier-learning algorithm via DCIFI projection pursuit ....................................................................... 286

11.2.3 Examples of the classification problems solved by the DCIFI projection classifier............................................................................ 290

11.3 Fuzzified Choquet Integral with Fuzzy-Valued Integrand (FCIFI) .................... 300

11.3.1 Definition of the FCIFI ................................................................... 300

11.3.2 The FCIFI with respect to monotone measures ..................................... 303

11.3.3 The FCIFI with respect to signed efficiency measures............................ 306

11.3.4 GA-based optimization algorithm for the FCIFI with respect to signed efficiency measures 


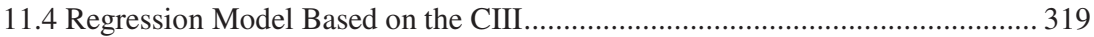

11.4.1 CIII regression model.................................................................... 319

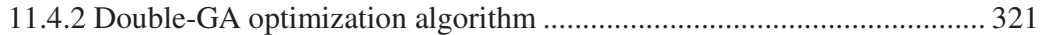

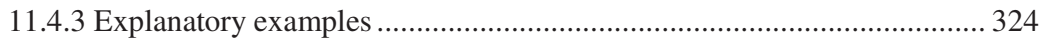

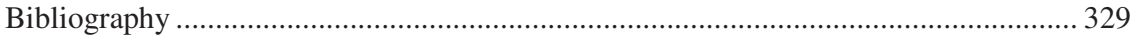

Index 


\section{List of Tables}

Table 6.1 Iris data (from ftp://ftp.ics.uci.edu/pub/machine-learning-databases) ........... 179

Table 6.2 Data of working times in Example 6.4 …...................................................... 183

Table 6.3 The scores of TV sets in Example 6.5 ........................................................... 184

Table 10.1 Data for linear classification in Example 10.1 ........................................... 241

Table 10.2 Artificial training data in Example 10.7 ....................................................... 252

Table 10.3 The preset and retrieved values of monotone measure $\mu$ and weights $b \ldots \ldots .259$

Table 10.4 Data and their projections in Example 10.8 ................................................. 266

Table 11.1 Preset and retrieved values of the signed efficiency measure and boundaries in Example 11.4 f.................................................................... 293

Table 11.2 Preset and retrieved values of the signed efficiency measure and boundaries in Example 11.5 ................................................................... 294

Table 11.3 The estimated values of the signed efficiency measure and the virtual boundary in two-emitter identification problem ...................................... 297

Table 11.4 Testing results on two-emitter identification problem with/without noise ... 298

Table 11.5 The estimated values of the signed efficiency measure and the virtual boundary in three-emitter identification problem .................................... 299

Table 11.6 Testing results on three-emitter identification problem with/without

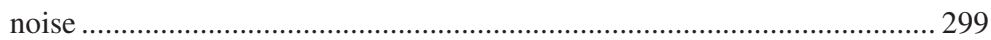

Table 11.7 Values of the signed efficiency measure $\mu$ in Example 11.13 ...................... 318

Table 11.8 Results of 10 trials in Example 11.14 ........................................................ 326

Table 11.9 Comparisons of the preset and the estimated unknown parameters of the best trial in Example 11.14 .................................................................... 326

Table 11.10 Results of 10 trials in Example 11.15 .................................................... 327

Table 11.11 Comparisons of the preset and the estimated unknown parameters of the best trial in Example 11.15 


\section{List of Figures}

Figure 1.1 The relation among chapters................................................................ 3

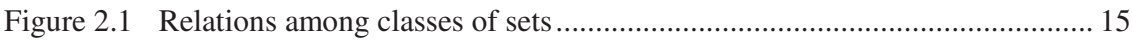

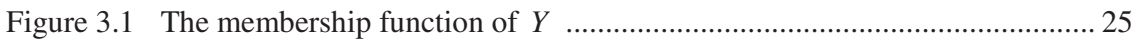

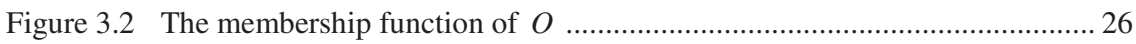

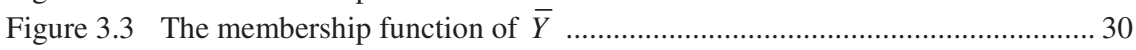

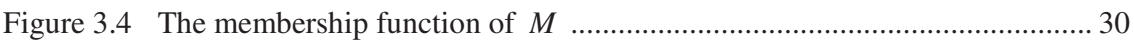

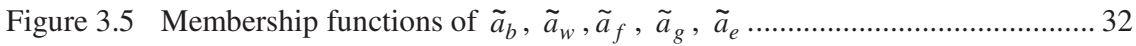

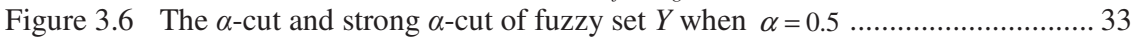

Figure 3.7 An $\alpha$-cut of convex fuzzy set with membership function $m(x)=e^{-x^{2}} \ldots \ldots \ldots . .38$

Figure 3.8 The membership function of $D+F$ obtained by the extension principle. ...... 43

Figure 3.9 The membership function of a rectangular fuzzy number ......................... 47

Figure 3.10 The membership function of a triangular fuzzy number........................... 49

Figure 3.11 The membership function of a trapezoidal fuzzy number.......................... 49

Figure 3.12 The membership function of a cosine fuzzy number. ................................ 50

Figure 3.13 Membership functions in Example 3.18 ................................................... 56

Figure 3.14 Membership functions in Example 3.19 ..................................................... 57

Figure 5.1 The geometric meaning of a definite integral .......................................... 125

Figure 5.2 The calculation of the Choquet integral defined on a finite set

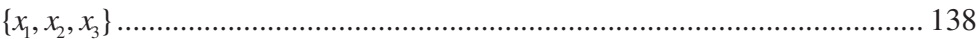

Figure 5.3 The chain used in the calculation of the Choquet integral in Example 5.7

Figure 5.4 The partition of $f$ corresponding to the Choquet integral in Example 5.17.

Figure 5.5 The partition of $f$ corresponding to the Lebesgue integral in

Example 5.18.

Figure 5.6 The partition of $f$ corresponding to the upper integral in Example 5.19.... 169

Figure 5.7 The partition of $f$ corresponding to the lower integral in Example 5.20 .... 170

Figure 5.8 The partitions corresponding to various types of nonlinear integrals in

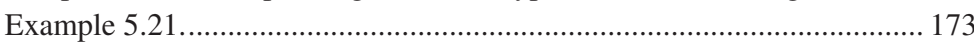

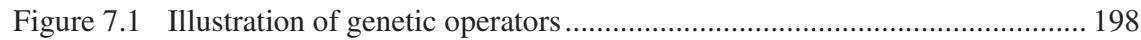

Figure 7.2 The flowchart of genetic algorithms .................................................... 198 
Figure 8.1 The lattice structure for the power set of a universal set with 4 attributes

Figure 10.1 The training data and one optimal classifying boundaries $x_{1}+2 x_{2}=1.4$ with a new sample $(0.3,0.7)$ in Example 10.1

Figure 10.2 Interaction between length and width of envelops in Example 10.2 .......... 243

Figure 10.3 The contours of the Choquet integral in Example 10.3 ............................. 244

Figure 10.4 The projection by the Choquet integral in Example 10.3 ........................ 245

Figure 10.5 A contour of the Choquet integral with respect to a signed efficiency measure in Example 10.4......

Figure 10.6 Contours of the Choquet integral with respect to a subadditive efficiency measure in Example 10.5.

Figure 10.7 Projection line and Contours of the weighted Choquet integral in Example 10.6.

Figure 10.8 View classification in Example 10.7 from three different directions.

Figure 10.9 The distribution of the projection $\hat{Y}$ on axis $L$ based on the training data set in Example 10.7.

Figure 10.10 The convergence of the genetic algorithm in Example 10.7 with different population sizes

Figure 10.11 Different projections share the same classifying boundary in Example 11.8

Figure 10.12 Two-class two-dimensional data set that can be well classified by cross-oriented projection pursuit

Figure 10.13 Two-class three-dimensional data set that can be well classified by cross-oriented projection pursuit.

Figure 11.1 The $\alpha$-level set of a fuzzy-valued function in Example 11.1 ...................... 275

Figure 11.2 A typical 2-dimensional heterogeneous fuzzy data .................................. 284

Figure 11.3 The DCIFI projection for 2-dimensional heterogeneous fuzzy data ........... 285

Figure 11.4 Illustration of virtual projection axis $L$ when determining the boundary of a pair of successive classes $C_{k_{i}}$ and $C_{k_{i+1}}$ : (a) when

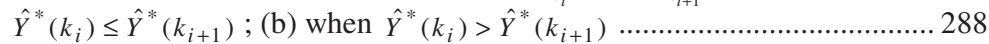

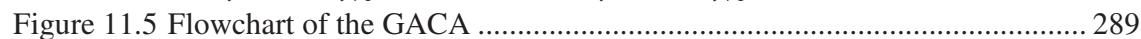

Figure 11.6 The training data and the trained classifying boundaries in Example 11.4.

Figure 11.7 Artificial data and the classification boundaries in Example 11.5 from two view directions

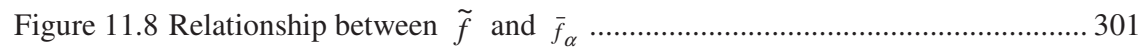

Figure 11.9 The membership functions and $\alpha$-cut function of $\tilde{f}$ in Example 11.6....... 302

Figure 11.10 The membership functions of the Choquet integral with triangular fuzzy-valued integrand in Example 11.7

Figure 11.11 The membership functions of the Choquet integral with normal fuzzyvalued integrand in Example 11.8

Figure 11.12 Description of terminal ranges when $\mu$ is a signed efficiency measure..... 308 
Figure 11.13 Correspondence in coding method ........................................................... 310

Figure 11.14 Distance definition on calculation of the left and the right terminals of

(C) $\int \bar{f} d \mu$

Figure 11.15 Membership functions of $\tilde{f}\left(x_{1}\right)$ and $\tilde{f}\left(x_{2}\right)$ in Example 11.11 .............. 315

Figure 11.16 The membership functions of (C) $\int \bar{f} d \mu$ in Example 11.11 .................... 315

Figure 11.17 Membership functions of $\tilde{f}\left(x_{1}\right)$ and $\tilde{f}\left(x_{2}\right)$ in Example 11.12...............316

Figure 11.18 The membership functions of (C) $\int \bar{f} d \mu$ in Example 11.12 .................... 317

Figure 11.19 Membership function of (C) $\int \bar{f} d \mu$ in Example 11.13............................ 318

Figure 11.20 Structure of an individual chromosome in the double-GA optimization

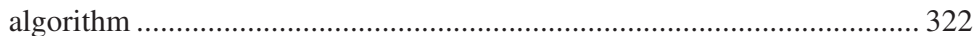

Figure 11.21 Benchmark model in Examples 11.14 and 11.15 ..................................... 325 